\title{
INVARIANT IDEALS AND BOREL SETS
}

\author{
ANDRZEJ PELC
}

\begin{abstract}
We investigate the size of the algebra $B(I)$, where $B$ is the family of Borel sets and $I$ is a translation invariant ideal of sets of reals. In particular the question whether $B(I)$ can contain Vitali selectors or even all sets of reals is discussed in connection with the completeness of $I$ and its invariance.
\end{abstract}

All our considerations refer to the set $\mathbf{R}$ of reals. $\mathbf{Q}$ denotes the set of rationals and $B$ the algebra of Borel sets. A family $I$ of subsets of a set $A$ is called an ideal on $A$ iff $I$ contains all singletons, does not contain $A$, and is closed under the operations of finite unions and of taking subsets. An ideal $I$ on $A$ is called uniform iff $X \in I$ whenever $|X|<|A|$ and is called $\kappa$-complete iff $\bigcup X \in I$ whenever $X \subset I$ and $|X|<\kappa ; \omega_{1}$-complete ideals are called $\sigma$-complete. An ideal $I$ on $\mathbf{R}$ is called invariant iff $x+A \in I$ for every $A \in I$ and $x \in \mathbf{R}$. It is called Q-invariant iff $x+A \in I$ for every $A \in I$ and $x \in \mathbf{Q}$. For any ideal $I$ on $\mathbf{R}, B(I)$ denotes the algebra of sets $\{B \triangle N: B \in B, N \in I\} . P(X)$ denotes the set of all subsets of $X$.

If $I$ is the ideal of Lebesgue measure zero sets or of meager sets then $B(I)$ does not contain Vitali selectors (selectors of the family of cosets of $(\mathbf{Q},+)$ in $(\mathbf{R},+)$ ). We investigate the problem if this remains true for more general classes of invariant or $\mathbf{Q}$-invariant ideals. We also discuss the possibility $B(I)=P(\mathbf{R})$ in this context.

Our first theorem shows that for some general classes of ideals the algebra $B(I)$ does not contain Vitali selectors or other specifically constructed sets.

THEOREM 1. (a) There exists a set $X$ of reals such that $X \notin B(I)$ for any $2^{\omega}$-complete invariant ideal I on $\mathbf{R}$.

(b) Assume $C H$. There exists a set $X$ of reals such that $X \notin B(I)$ for any $\sigma$ complete $\mathbf{Q}$-invariant ideal I on $\mathbf{R}$.

(c) Assume $C H$ and Kurepa's hypothesis. For every $\sigma$-complete $\mathbf{Q}$-invariant ideal $I$ there exists a Vitali selector $S \notin B(I)$.

Proof. (a) Let $H=\left\{h_{\alpha}: \alpha<2^{\omega}\right\}$ be a Hamel basis and $S_{\alpha}$ the set of those reals in whose $H$-representation the elements $h_{\beta}: \beta<\alpha$ do not appear at all and the element $h_{\alpha}$ appears with coefficient 1 . Hence those sets are pairwise disjoint and $S_{\alpha}$ is a selector of the family of cosets of the group generated by $\left\{h_{\beta}: \beta \leq \alpha\right\}$. Let $\left\{B_{\alpha}: \alpha<2^{\omega}\right\}$ be an enumeration of $B$. We define $T_{\alpha}=S_{\alpha} \backslash B_{\alpha}$ and $X=\bigcup_{\alpha<2^{\omega}} T_{\alpha}$. For any $\alpha<2^{\omega}$ we get

$$
X \triangle B=\left(X \backslash B_{\alpha}\right) \cup\left(B_{\alpha} \backslash X\right) \supset T_{\alpha} \cup\left(B_{\alpha} \cap S_{\alpha}\right)=S_{\alpha} .
$$

If $I$ is a $2^{\omega}$-complete invariant ideal the sets $S_{\alpha}$ are outside of $I$, which shows that $X \notin B(I)$.

Received by the editors October 10, 1984 and, in revised form, July 11, 1985.

1980 Mathematics Subject Classification. Primary 04A20, 03E05; Secondary 28 A05. 
(b) Take any uncountable family of pairwise almost disjoint functions $f: \omega_{1} \rightarrow$ $\omega$. It gives also an uncountable family $\left\{S_{\alpha}: \alpha<\omega_{1}\right\}$ of Vitali's selectors. Let $\left\{B_{\alpha}: \alpha<\omega_{1}\right\}$ be an enumeration of $B$. We construct sets $\left\{T_{\alpha}: \alpha<\omega_{1}\right\}$ by putting

$$
T_{\alpha}=\left(S_{\alpha} \backslash B_{\alpha}\right) \backslash \bigcup_{\beta<\alpha} S_{\beta} .
$$

Let finally $X=\bigcup_{\alpha<\omega_{1}} T_{\alpha}$. Hence, for any $\alpha<\omega_{1}$

$$
X \triangle B_{\alpha}=\left(X \backslash B_{\alpha}\right) \cup\left(B_{\alpha} \backslash X\right) \supset T_{\alpha} \cup\left(B_{\alpha} \cap S_{\alpha}\right) \supset S_{\alpha} \backslash \bigcup_{\beta<\alpha} S_{\beta} .
$$

Take any $\sigma$-complete $\mathbf{Q}$-invariant ideal $I$. Hence $S_{\alpha} \notin I$ for $\alpha<\omega_{1}$ and since the set $S_{\alpha} \cap \bigcup_{\beta<\alpha} S_{\beta}$ is countable, this shows that $X \triangle B_{\alpha} \notin I$ for any $\alpha<\omega_{1}$. Hence $X \notin B(I)$.

(c) By Kurepa's hypothesis there exists a family of $\omega_{2}$ pairwise almost disjoint functions $f: \omega_{1} \rightarrow \omega$. In view of $\mathrm{CH}$ this leads to a family $\left\{S_{\alpha}: \alpha<\omega_{2}\right\}$ of pairwise almost disjoint Vitali selectors. Let $I$ be any $\sigma$-complete $\mathbf{Q}$-invariant ideal on $\mathbf{R}$. Then $\forall \alpha<\omega_{2}, S_{\alpha} \notin I$ and $\forall \alpha, \beta<\omega_{2} \alpha \neq \beta \Rightarrow S_{\alpha} \cap S_{\beta} \in I$. Since by CH $\omega_{2}>|B|$, this shows that for some $\alpha<\omega_{2}, S_{\alpha} \notin B(I)$.

The next theorem shows that Vitali selectors may belong to $B(I)$ or even to $I$ for some translation invariant ideals.

THEOREM 2. (a) There exists a $2^{\omega}$-complete $\mathbf{Q}$-invariant ideal I such that the algebra $B(I)$ contains a Vitali selector.

(b) There exists an invariant ideal I containing all Vitali selectors.

Proof. (a) It is enough to construct a nonempty perfect set $P$ such that $q+P$ is disjoint from $P$ for every $q \in \mathbf{Q}$. Then $P$ is a subset of a Vitali selector $S$ and the set $\bigcup_{q \in \mathbf{Q}} q+(S \backslash P)$ generates a $2^{\omega}$-complete $\mathbf{Q}$-invariant ideal $I$ such that $S \in B(I)$.

We construct the set $P$ by induction simultaneously defining a function $f: \omega \rightarrow \omega$ and a family of closed sets $\left\{A_{n}: n \in \omega\right\}$. In the first step we chose the interval $A_{1}=[0,1]$ and let $f(1)=1$. Suppose that $2^{n-1}$ closed subintervals of $[0,1]$ of length $1 / f(n)$ were chosen in the $n$th step and their union was called $A_{n}$. In the $(n+1)$ step we divide each of those intervals into $(n+1)$ equal closed subintervals and take the coinitial one in each case. Then we divide each of thus obtained $2^{n-1}$ intervals $I_{1}, \ldots, I_{2^{n-1}}$ of length $1 / f(n)(n+1)$ into $2^{n}-1$ equal closed subintervals $I_{i}^{1}, \ldots, I_{i}^{2^{n}-1}, 1 \leq i \leq 2^{n-1}$. Finally we choose $2^{n}$ intervals $I_{1}^{1}, I_{1}^{3}, I_{2}^{5}, I_{2}^{7}, \ldots, I_{i}^{4 i+1}, I_{i}^{4 i+3}, \ldots, I_{2^{n-1}}^{2^{n}-3}, I_{2^{n-1}}^{2^{n}-1}$ and call their union $A_{n+1}$.

The descending closed sets $A_{n}: n \in \omega$ are now defined by induction. We put $P=\bigcap_{n \in \omega} A_{n} . P$ is clearly perfect and $(q+P) \cap P=\varnothing$ for any $q \in \mathbf{Q}$ because in the $n$th step we assured that $\left(k / n f(n-1)+A_{n}\right) \cap A_{n}=\varnothing$ for any $1 \leq k \leq n f(n-1)$.

(b) Let $S$ be a Vitali selector and $x_{1}, \ldots, x_{k} \notin \mathbf{Q}, q_{1}, \ldots, q_{k} \in \mathbf{Q}$. Consider any coset $x+\mathbf{Q}$, where $x \in S$. We show that the set $A=\left(\left\{x_{1}, \ldots, x_{k}, q_{1}, \ldots, q_{k}\right\}+S\right) \cap$ $(x+\mathbf{Q})$ is finite. Indeed suppose $x_{1}+s_{1}=x+q_{1}, x_{1}+s_{2}=x+q_{2}$, where $s_{1}, s_{2} \in S$. Hence $s_{1}-s_{2}=q_{1}-q_{2} \in \mathbf{Q}$, which implies $s_{1}=s_{2}$. This shows that $A$ has at most $2 k$ elements. Hence for any finite family $\left\{S_{1}, \ldots, S_{n}\right\}$ of Vitali selectors and any finite set $C$ of reals the set $\bigcup_{i=1}^{n} C+S_{i}$ has a finite intersection with every coset of $(\mathbf{Q},+)$ in $(\mathbf{R},+)$. This implies that the family of all Vitali selectors generates an invariant ideal. 
The above results show that the degree of completeness and of invariance enjoyed by an ideal $I$ may have major impact on the size of $B(I)$. We will now discuss the question whether $B(I)$ may contain all sets of reals for $\mathbf{Q}$-invariant or invariant ideals $I$, gradually restricting attention to more and more complete ones.

If no completeness is assumed then $I$ can be a prime ideal and hence $B(I)$ contains all subsets of $\mathbf{R}$. In this case we can also require that $I$ be uniform. If we restrict attention to $\mathbf{Q}$-invariant ideals, they cannot be prime anymore (see $[\mathbf{3}$, Remark 3]) but may have the property $B(I)=P(\mathbf{R})$. It suffices to take the ideal generated by singletons and $\mathbf{R} \backslash \mathbf{Q}$. However this ideal is not uniform (cf. Problem 1 ). The problem if $B(I)$ !ray contain all sets of reals for some invariant ideal $I$ remains open (Problem 2).

We next turn attention to $\sigma$-complete ideals. In the $\mathbf{Q}$-invariant setting the existence of such ideals $I$ for which $B(I)$ contains all sets of reals is independent of ZFC.

PROPOSITION 3. The statement: "There exists a $\sigma$-complete $\mathbf{Q}$-invariant ideal I for which $B(I)=P(\mathbf{R})$ " is independent of $Z F C$.

Proof. Assuming the continuum hypothesis, for every $\sigma$-complete ideal $I$ on $\mathbf{R}$ there is a family $\left\{X_{\alpha}: \alpha<\omega_{1}\right\}$ of pairwise disjoint sets outside of $I$ (because $I$ cannot be $\sigma$-saturated). This shows that the algebra $P(\mathbf{R}) / I$ has cardinality $2^{\omega_{1}}$ hence greater than $2^{\omega}$, which implies $B(I) \neq P(\mathbf{R})$. On the other hand a lemma of Silver (cf. Martin and Solovay [1]) says that if Martin's Axiom (MA) is assumed and $A$ is a set of reals of cardinality $<2^{\omega}$ then for every $C \subset A$ there is a Borel set $C^{*}$ s.t. $C=C^{*} \cap A$. Assume $\left.\mathrm{MA}+\right\rceil \mathrm{CH}$ and let $A$ be any union of $\omega_{1}$ cosets of $(\mathbf{Q},+)$ in $(\mathbf{R},+)$. Then the $\sigma$-complete ideal $I$ generated by $\mathbf{R} \backslash A$ and all singletons is $\mathbf{Q}$-invariant and $B(I)=P(\mathbf{R})$.

REMARK. The ideal $I$ constructed above is not uniform but those ideals are excluded by the following result of Taylor $[\mathbf{4}]:|P(\mathbf{R}) / I|>2^{\omega}$ for all uniform $\sigma$ complete ideals $I$ on $\mathbf{R}$.

Further restriction to $\sigma$-complete invariant ideals prohibits $B(I)$ from containing all sets of reals.

Proposition 4. For every $\sigma$-complete invariant ideal $I, B(I) \neq P(\mathbf{R})$.

ProOF. We follow an argument from Pelc [2]. Let $\left\{h_{\alpha}: \alpha<2^{\omega}\right\}$ be a Hamel basis and for any finite sequence $s=\left(q_{0}, \ldots, q_{n}\right)$ of rationals let

$$
V_{s}=\left\{q_{0} h_{\alpha_{0}}+\cdots+q_{n} h_{\alpha_{n}}: \alpha_{0}<\cdots<\alpha_{n}<2^{\omega}\right\}
$$

By $\sigma$-completeness of $I, V_{s_{0}} \notin I$ for some finite sequence $s_{0}$. Let $q$ be any rational which does not appear in $s_{0}$. Clearly for any $\alpha<\beta<2^{\omega}$

$$
\left(q h_{\alpha}+V_{s_{0}}\right) \cap\left(q h_{\beta}+V_{s_{0}}\right)=\varnothing .
$$

By invariance of $I$ we get a family of $2^{\omega}$ pairwise disjoint sets outside of $I$. This shows that the cardinality of the algebra $P(\mathbf{R}) / I$ is $2^{2^{\omega}}$ and hence $B(I) \neq P(\mathbf{R})$.

Let us finally remark that for $2^{\omega}$-complete ideals $I, B(I)$ cannot contain all sets of reals even if no invariance is assumed. This follows from Taylor's result mentioned above, since $2^{\omega}$-complete ideals are uniform.

We close the paper with a list of open problems. Two of them were mentioned before. 
Problem 1. Does there exist a uniform Q-invariant ideal $I$ such that $B(I)=$ $P(\mathbf{R})$ ?

Problem 2. Does there exist an invariant ideal $I$ such that $B(I)=P(\mathbf{R})$ ?

The third problem is due to Brzuchowski and Cichon [0] (unpublished). A partial solution to it is given in Theorem 2(a).

Problem 3. Does there exist a $\sigma$-complete invariant ideal $I$ such that $B(I)$ contains a Vitali selector?

\section{REFERENCES}

0. J. Brzuchowski and J. Cichoń, Miara $i$ kategoria, unpublished.

1. D. A. Martin and R. M. Solovay, Internal Cohen extensions, Ann. of Math. Logic 2 (1970), 143-178.

2. A. Pelc, Invariant measures and ideals on discrete groups, Dissertationes Math. (in print).

3. C. Ryll-Nardzewski and R. Telgársky, The nonexistence of universal invariant measures, Proc. Amer. Math. Soc. 69 (1978), 240-242.

4. A. Taylor, On the cardinality of the algebra $P(\kappa) / I$, handwritten notes.

Département D'INFormatique, Université du Québec À Hull, Case Postale 1250, SuCCURSALE B, HULL, QUÉBEC J8X 3X7, CANADA 